\title{
Translational research in psychiatry: pitfalls and opportunities for career development
}

\author{
Molecular Psychiatry (2004) 9, 117. doi:10.1038/ \\ sj.mp.4001479
}

Translational research is at the forefront of contemporary biomedical science. As immense progress has been made in basic research, the challenge is now to translate fundamental discoveries from the arena of pure science to the reality of health care, leading to better preventive approaches, more refined and successful treatments, and improved health. The translational approach is self-evident in the field of oncology. One can study cancerous cells in cultures, use animal models that develop cancer spontaneously, induce cancer by chemicals or radiation, and transfer human tumors to animals. Using those models, the tools of molecular biology can contribute to elucidate the mechanisms of neoplastic transformation as well as local and distant tumor growth and suppression. Once fundamental mechanisms are better understood and new insights are gained into diagnosis and treatment, those can then be translated to clinical investigation.

If only things were so straightforward in psychiatry! Psychiatric disorders affect higher cognitive functions and complex affective states that differentiate us from other animal species. We justify animal experimentation by claiming that animals lack precisely those higher cognitive functions and complex affective states that are dysregulated in psychiatric disorders. It is therefore tautological to propose that animal models can completely reproduce a psychiatric disorder-at best they can offer tools for the study of the biological substrates of specific features of a psychiatric diagnosis. The cells whose functions are presumably affected-neurons-are terminally differentiated and do not grow well in culture. Moreover, the organ of interest is locked inside an osseous cavity that makes it inaccessible. Finally, the fundamental biological processes underlying psychiatric disorders are unknown and consequently not amenable to modeling in the laboratory. In the light of these severe conceptual limitations, how can translational approaches be applied to the field of psychiatry?

A useful starting point that goes beyond the limitations discussed above is offered by the emerging disciplines of genomics and pharmacogenomics. A key limitation in psychiatry now is the absence of exciting new drugs that exert their therapeutic effects by targeting pathways and circuits identified by the tools of contemporary biology. Identification of novel therapeutic targets for drug development is therefore important and necessary. The sequencing of the human genome and the use of high throughput technologies make it possible to search for candidates that go beyond our tired monoaminergic targets. These technical developments have not yet been fully incorporated into the field of psychiatry. Even when they are, they have unique technical issues that have to be understood and dealt with effectively.

Traditional research queries one system at a time in a narrow, hypothesis-driven approach. The hypotheses are now much broader and sometimes even unnecessary. Rather than postulating that a specific event will happen and test that, why not query the entire genome, proteome, or metabolome, and ascertain all changes that can happen? Formerly called 'fishing expedition', these hypotheses-generating experiments may open the doors to new and unexpected targets, which we badly need, by going beyond our pre-conceived ideas and theories. However, as the number of genes, proteins, and metabolites that we can query far exceeds the number of samples available in any single study, how does one deal statistically with a situation in which the number of tests exceeds the size of study groups? How is that kind of approach validated and how can divergent results be interpreted when multiple techniques are used for confirmation and replication?

Importantly, as we take advantage of high throughput approaches, who will put together, finance, manage, and lead the complex multidisciplinary groups that are needed to master the technical complexity of genomics, proteomics, and metabolomics, the intricacy of the necessary laboratory validation methods, and the ability to rigorously test new candidates in clinical research studies? At present, little is being done to generate a cadre of highly qualified physician investigators who can effectively lead multidisciplinary translational research groups in psychiatry that integrate genomic science, molecular neurobiology, and clinical investigation. Academic health centers and their departments of psychiatry should prioritize among their multiple missions the professional development of outstanding academic leaders in translational psychiatry. While it is truly essential to increase the number of entry-level, patient-oriented investigators, it is equally critical to foster the professional development of the next generation of effective mid-career and senior academic leaders who can transform translational investigation from the incipient cottage industry it is today into the keystone of biomedical science.

J Licinio* and M-L Wong UCLA-NPI Center for Pharmacogenomics licinio@ucla.edu 